\title{
Foscolo e Leopardi: poetiche a confronto Andréia Guerini
}

\begin{abstract}
L'analisi e il confronto di alcuni aspetti delle poetiche di Foscolo e Leopardi permettono di affermare che la poetica di Leopardi è più universale, in quanto riguarda gli aspetti estetici, che vanno oltre il loro tempo. A sua volta, la poetica di Foscolo è più nazionale, perché riguarda la politica e gli aspetti sociali della sua epoca. PAROLE CHIAVE: letteratura italiana; Ugo Foscolo; Giacomo Leopardi.
\end{abstract}

Introduzione

Dopo una lunga paralisi o, per usare le parole di Carpeaux, una serie di "intermitências" o "renascenças sucessivas", solo nel secolo XIX la letteratura italiana ha riacquistato una posizione di rilievo nello scenario internazionale. Come osserva Carpeaux:

Dante, o Juiz, é o mestre de toda a literatura italiana. Seguem-se-the Petrarca [...]; Ariosto; Alfieri, o homem de ferro [...]; Foscolo, o paeta do exilio; e enfim Leopardi. Manzoni e Carducci representam o fim das tradiçóes que criaram a Itália moderna [...] depois deles, é o vácuo. (1999: 219)

L'assenza evocata in questo brano non è solo una caratteristica del postromanticismo: anche tra il Seicento e l'Ottocento è possibile riscontrare un vuoto nella produzione letteraria italiana. Questo fatto è stato sottolineato da Leopardi quando, in un frammento dello Zibaldone di Pensieri (1817-1832), nelle vesti di storico della letteratura, afferma: 
Il trecento fu il principio della nostra letteratura, non già il colmo, imperocchè non ebbe se non tre scrittori grandi: il quattrocento non fu corruzione nè [2] raffinamento del trecento, ma un sonno della letteratura (che avea dato luogo all'erudizione) la quale restava ancora incorrotta e peccava ancora più tosto di poco. Poliziano, Pulci. Il cinquecento fu vera continuazione del trecento $\mathrm{e}$ il colmo della nostra letteratura. Di poi venne il raffinamento del seicento, che nel settecento s'è solamente mutato in corruzione d'altra specie, ma il buon gusto nel volgo dei letterati non è tornato più, nè tornerà secondo me, perché dal niente si pud passare al buono, ma dal troppo buono o sia dal corrotto stimo che non si possa'.

Nonostante Leopardi affermi che "dal niente si può passare al buono, ma dal troppo [...] stimo che non si possa", è possibile dire che nell'Ottocento l'Italia, grazie a Leopardi (1798-1837) e Foscolo (1778-1827), ha contribuito ad arricchire quella che Hobsbawn ha definito l'“era de superlativos"2 europea.

In uno dei suoi primi saggi, Borges sostiene che:

Hay escritores soslayados y chúcaros (Swinburne, Evaristo Carriego, Rafael Cansinos-Assens) cuya total aventura humana es la de su obra; hay otros de vida cargada, cuya escritura es apenas un rato largo, un episodio de sus pobladísimos días. (1997:130)

Seguendo questo ragionamento, mi sembra che Foscolo possa essere incluso nella seconda categoria descritta da Borges, e Leopardi nella prima, come vedremo in seguito.

1. I frammenti qui usati sono stati tratti dall'edizione integrale dello Zibaldone di Pensieri disponibile nel sito internet: http://www.liberliber.it/biblioteca/l/leopardi/ del 02/02/02.

2. Nel campo politico e sociale, quest'affermazione è valida fino al 1840. Dopo, secondo Hobsbawn, "deixa-se - mundo dos superlativos para se entrar no mundo das afirmaçóes modestas" ( $A$ era das revoluçōes - Europa 1789-1848, 2001: 321-3). La stessa cosa sembra succedere nella storia della letteratura e della critica. Wellek afferma che "the years around 1830 brought a deep break in literary history and in the history of criticism. A great generation passed away: in Germany Friedrich Schlegel (1829), Hegel (1831) e Goethe (1832) died in quick succession; in England Hazlitt (1830), Coleridge (1834) and Lamb (1834); in Italy Foscolo (1827) and Leopardi (1837). Those who survived fell silent, at least as critics" (1970: 335). 
Cenni biografici e teorici

Foscolo fu un uomo "rivolto verso il mondo esterno"; viaggiò molto, si autoesiliò, visse in Francia, Inghilterra e Svizzera. Ebbe una vita molto agitata, di "vizii ricca e di virtù", come lui stesso ${ }^{3}$ la definl: amori tumultuosi, intensa partecipazione politica ecc. Come afferma De Rienzo,

la vita di Foscolo è dunque caratterizzata dal gusto delle passioni intense, dagli amori tumultuosi, da solenni giuramenti in nome della libertà, da rotture clamorose, da gesti comunque spettacolari. Per questo Foscolo incarnò, per le generazioni avvenire, [...], il mito del "libero scrittore" e la sua vita divenne un modello di vita romantica" (1997: 145)

R. Ceserani e L. de Federicis descrivono Foscolo come "prodigo, megalomane, incline alle polemiche, sempre impetuosamente innamorato" (1993: 81). Impegnato nella causa rivoluzionaria e nella difesa dell' indipendenza nazionale, Foscolo è stato un personaggio del suo tempo.

A sua volta, Leopardi fu un uomo "rivolto verso il mondo interiore", cioè verso la sua Recanati, o il "borgo selvaggio", come lui stesso la chiamava. Ebbe problemi con la famiglia, pochi amici, nessun amore corrisposto e ancora, come sottolinea Karl Vossler, era "enfermo de alma y cuerpo" (1951: 155). Per motivi legati a conflitri con la situazione egemonica del momento, Leopardi non fu un uomo del suo tempo; fu un moderno avant la lettre in molti sensi, poiché anticipa, in diversi campi, aspetti della modernità, specialmente nello Zibaldone di Pensieri.

In termini biografici Foscolo e Leopardi si differenziano, ma presentano alcune analogie, soprattutto per quanto riguarda la formazione letteraria: entrambi sono eruditi che vissero nell'isolamento degli studi. Si dedicarono allo studio dei classici greci, latini e italiani ed anche ad autori moderni italiani e stranieri. Sia Foscolo che

3. Eugenio Donadoni, "Biografia di Foscolo" nell'edizione elettronica di Giuseppe Bonghi del 20/04/1999 in: http://www.fausernet.novara.it/fauser/biblio/index 130.htm, Progetro Ugo Foscolo del 01/03/02. 
Leopardi furono testimoni di un importante e effervescente momento della storia culturale, politica e sociale dell'Italia e dell'Europa.

Foscolo, che, secondo De Sanctis, chiuse 'il suo secolo co' Carmi Dei Sepolcri e Le Grazie", e Leopardi, che fu "il principale poeta dei tempi moderni" (1986: 1031), tolsero la letteratura italiana da un silenzio durato quasi due secoli, facendo sì che l'Italia uscisse dalla condizione periferica alla quale era stata relegata, dopo essere stata, nel Rinascimento, all'avanguardia.

Aspetti di due poetiche

Sia Foscolo che Leopardi hanno una produzione letteraria molto varia e intensa, che comprende generi e campi d'interventi culturali molto diversi. Sono stati, ad esempio, traduttori. Tradurre e parafrasare poeti antichi è stata una delle prime attività di entrambi. Foscolo ha tradotto alcuni frammenti dell' Iliade di Omero, la Chioma di Berenice di Callimaco, Viaggio sentimentale di Yorick lungo La Francia e l'Italia (1813) di Sterne, ecc. Leopardi ha tradotto il quinto idillio di Mosco, la Batracomiomachia dello pseudo-Omero, la Titanomachia di Esiodo, il secondo libro dell'Eneide e il primo dell'Odissea. Oltre che traduttori, sono stati entrambi critici della traduzione.

La posizione di Foscolo e Leopardi sul concetto di traduzione è diversa. Foscolo ritiene che il traduttore debba penetrare e esprimere al massimo lo spirito dell'originale, avvicinandosi cos̀ al progetto romantico tedesco, che si schierò a favore della "fedeltà" nelle traduzioni. Per Leopardi invece una buona traduzione è quella che riesce a trovare la sintesi tra il carattere nazionale e lo straniero, cioc̀ la mediazione nella conservazione di elementi del testo di partenza nel testo d'arrivo ${ }^{4}$.

Oltre ad essere tradurtori e critici, Leopardi e Foscolo sono celebrati come poeti, meno ammirati come prosatori e quasi sconosciuti come saggisti. E da

4. Per ulteriori informazioni sulla concezione di traduzione di Leopardi vedi Guerini, Andréia. A poética de Leopardi: gênero e traduçâo no Zibaldone di Pensieri, Florianópolis, UFSC, 2001. Tesi di dottorato. 
questo insieme che possiamo estrarre e confrontare alcuni elementi della loro poeticas.

Se si pensa agli scritri di Leopardi, si vedrà che lo Zibaldone di Pensieri con le sue 4.526 pagine manoscritte $\dot{e}$ il luogo privilegiato della sua elaborazione poetica. Leopardi ha scritto altri saggi, come la "Lettera ai compilatori della 'Biblioteca italiana' in risposta a quella di Madama la baronessa di Stäel" (1816) e il "Discorso intorno alla poesia romantica" (1818). Oltre che in questi saggi, non possiamo dimenticare che anche nell'intenso epistolario di Leopardi è possibile trovare elementi della sua poetica.

Probabilmente a causa della sua vita conturbata, la formulazione poetica di Foscolo è meno ampia, però non meno importante, anche se Wellek afferma che, per quanto riguarda i suoi saggi critici,

the fragmentariness and incompleteness, the heavy admixture of patriotic oratory and inerte obsolete antiquarianism, are not the only causes of disappointment. It is rather a certain lack of coherence and sharpness in the choice of ideas which make Foscolo an eclectic, a figure of transition who, however great his importance in the history of Italian criticism, will never acquire great stature in a European context. One could even make a case for Foscolo's criticism as a repertory of neoclassical commonplaces. (1970: 267)

È possibile sostenere che la poetica di Foscolo non si sviluppa solo nei suoi saggi critici, come vedremo dopo, ma si concentra nel suo epistolario e anche nei suoi carmi Dei Sepolcri (1806) e Le Grazie. Nel primo carme definì ed espresse la sua idea di poesia, principalmente nell'ultima parte del poema. Per Francesco Spera,

5. Il termine poetica è qui in teso e utilizzato come "teoria sistematica della poesia ${ }^{35}$, che mira a definire la natura della poesia, il suo tipo e la sua forma, ed i principi che la governano. O ancora, come afferma Walter Binni: 'con la parola 'poetica' si vogliono essenzialmente indicare la consapevolezza critica che il poeta ha della propria natura artistica, il suo ideale estetico, il suo programma, i modi secondo i quali si propone di costruire' (1993: 89). Per Emil Staiger, invece, “a Poética ensina em que consiste a essência da poesia; ordena os modelos existentes e com isso cria o problema do gênero; orienta os inexperientes que pretendem ocupar-se com a atividade poética" (1975: 181). 
"nell'ultima parte si concentra un'altissima meditazione sulla poesia" (Squarotti, 1991: 393). È da questa parte che trascrivo alcuni versi:

$[\ldots]$

E me che i tempi ed il desio d'onore

fan per diversa gente ir fuggitivo,

me ad evocar gli eroi chiamin le Muse

del mortale pensiero animatrici.

[...]

E tu onore di pianti, Ettore, avrai, ove fia santo e lagrimato il sangue per la patria versato, e finché il Sole risplenderà su le sciagure umane.

In questi versi è possibile intravedere la sostanza dell'esistenza stessa della poesia che si apre con la figura di Foscolo, che "i tempi e il desio d'onore / fan per diversa gente ir fuggitivo" (tema dell'esilio) e si chiude con la figura di Ettore, che muore per la difesa della patria: "e tu onore di pianti, Ettore, / avrai ove fia santo e lagrimato il sangue / per la patria versato" Secondo Mario Pazzaglia, l'ultima parte del carme

è un inno alla poesia, il cui compito è quello di tramandare non solo il ricordo degli eroi, ma anche i valori che essi affermarono. Essa, in tal modo, crea e diffonde il culto delle più alte illusioni che riscattano la nostra vita dal nulla. Quello del poeta diventa un sacerdozio altissimo di umanità e di civiltà: $\mathrm{e}$ la poesia diventa mezzo di suprema elevazione, di armonia spirituale e morale, di autentica civiltà ${ }^{6}$.

Si può affermare che i versi dei Sepolcri contengono un manifesto di poetica, o la sua "giustificazione poetica", reiterato, sia nel 1809, nell'introduzione al corso di eloquenza presso l'Università di Pavia, intitolato Dell'origine e dell'ufficio della letteratura, sia nella famosa lettera indirizzata a Monsieur Guillon, nella quale osserva:

6. Vedere Pazzaglia, Mario, Letteratura italiana. L'Ottocento. Testi e critica con lineamenti di storia letteraria, v. 3, Bologna, Zanichelli, 1991, pp. 68-69. 
[...] quantunque gli uomini di egregia virtù siano perseguitati vivendo, e il tempo distrugga $i$ lor monumenti, la memoria delle virtù e de' monumenti vive immortale negli scrittori, e si rianima negl'ingegni che coltivano le muse?.

Oltre a questo, per De Sanctis, i Sepolcri stabilirono la reputazione di Foscolo “e lo alzarono accanto a' sommi. $\mathrm{E}$, in verità, questo carme è la prima voce lirica della nuova letteratura [...]" (1996: 781).

Nel poema incompiuto Le Grazie, che secondo Carpeaux è il "mais clássico e mais pagão" (1961: 1595), si mostra invece dominante la tesi della funzione rasserenatrice dell'arte; o ancora, secondo The Cambridge History of Italian Literature, in questo poema, dedicato a Antonio Canova, "the highest achievement of Foscolo's new mythology is his celebration of the civilizing function of literature and art in general" (1999: 414). In questo carme è presente una notevole esaltazione della Bellezza, come è possibile osservare nel primo inno, dedicato a Venere:

Cantando, o Grazie, degli eterei pregi

Di che il cielo v'adorna, e della gioja

Che vereconde voi date alla terra,

Belle vergini! a voi chieggo l'arcana

Armoniosa melodia pirtrice

Della vostra beltà; sì che all'Italia

Afflitta di regali ire straniere

Voli improvviso a rallegrarla il carme.

[...]

Vieni, o Canova, e agl'inni. Al cor men fece

Dono la bella Dea che in riva d'Arno

Sacrasti alle tranquille arti custode;

Ed ella d'immortal lume e d'ambrosia

La santa immago sua tutta precinse.

Forse (o ch'io spero!) artefice di Numi,

7. Apud Bonghi, Giuseppe, "Introduzione ai Sepolcri di Ugo Foscolo" in http://www.fausernet.novara.it/ del $01 / 03 / 02$. 
Nuovo meco darai spirto alle Grazie

Ch'or di tua man sorgon dal marmo. Anch'io

Pingo e spiro a' fantasmi anima eterna:

Sdegno il verso che suona e che non crea;

Perché Febo mi disse: Io Fidia primo

Ed Apelle guidai con la mia lira.

In "Ragione Poetica del Carme", Foscolo ci ricorda che in questo carme "l'autore ebbe tre intenti diversi, i quali unitamente concorrono al fine essenziale della poesia, di ammaestrare dilettando"s. Qui il poeta si avvicina alle concezioni di Orazio, per il quale la poesia dev'essere "utile" e "dulce"

In alcuni dei suoi più importanti sonetti, come Alla sera, $A$ Zacinto, In morte del fratello Giovanni, Alla Musa e $A$ se stesso, è possibile individuare le riflessioni di Foscolo sulla poetica dell'esilio', come possiamo vedere nella terza strofa di $A$ se stesso:

Figlio infelice, e disperato amante,

E senza patria, a tutti aspro e a te stesso,

Giovine d'anni e rugoso in sembiante.

Oppure nella prima quartina di In morte del fratello Giovanni:

Un di, s'io non andrò sempre fuggendo

Di gente in gente, me vedrai seduto

Su la tua pietra, o fratel mio, gemendo

Il fior de' tuoi gentili anni caduto.

Le concezioni poetiche di Foscolo sono inoltre presenti nei suoi diversi saggi di critica letteraria, soprattutto nel "Saggio sullo stato della letteratura italiana nel

8. Vedere Foscolo, Ugo, "Ragione Poetica del Carme", in Frammenti abbozzati in http://www.fausernet.novara. it/ dell'11/03/02.

9. La poetica dell'esilio è anche presente in Le ultime lettere di Jacopo Ortis, nei Sepolcri, ne Le Gnazie e nell' Epistolario. 
primo ventennio del secolo decimo nono" (1818), nel quale Foscolo parla di Dante, Petrarca, Boccaccio, di poemi narrativi, della scuola drammatica contemporanea ecc. Questi saggi, per Salinari e Ricci,

hanno grande rilievo per l'elaborazione della poetica personale dell'autore, delle sue idee sull'arte, del suo gusto di scrittore e di lettore e per valutare l'apporto foscoliano all'inizio di una critica letteraria veramente moderna nella quale fosse presente, nello stesso tempo, un vivo senso storico e un'acuta capacità d'individuazione psicologica e linguistica. (1986: 139)

Si può dire che gli scritti critici di Foscolo si sviluppano in forma discontinua e disorganica, senza un metodo preciso, e questo si potrebbe far risalire alla mancanza di una formazione filosofica più solida. Nel contempo questi scritti introducono delle osservazioni che vanno dalla psicologia dell'autore all'analisi del linguaggio, o ancora dal rapporto tra la vita e l'opera, come nel saggio "Sopra l'amore di Petrarca", alla natura dell'arte, presente in "Dell'origine e dell' ufficio della letteratura" In questo saggio, Foscolo analizza e dà enfasi alla materia prima della letteratura, cioè alla parola: "ogni uomo sa che la parola è mezzo di rappresentare il pensiero; ma pochi si accorgono che la progressione, l'abbondanza e l'economia del pensiero sono effetti della parola" (1995: 509). La parola sta all'origine e sarà l'elemento principale della funzione della letteratura. Sottolinea Foscolo che "senza la facoltà della parola, le potenze mentali dell'uomo giacerebbero inerti e mortificate, ed egli privo di mezzi di comunicazione necessarj allo stato progressivo di guerra e di società, confonderebessi con le fiere" (1995: 518).

Lintenso interesse per la parola è evidente in alcuni passaggi dei saggi su Dante e Petrarca. In essi, Foscolo discute l'effetto prodotto dalle parole isolate. Questa preoccupazione si nota anche nelle sue analisi delle traduzioni di Omero in italiano fatte da altri scrittori, o di Tasso in inglese, le quali dimostrano la sua grande passione per la filologia.

Secondo Vincenzo de Caprio e Stefano Giovanardi,

in tutta la sua attività di letterato, Foscolo senti la necessità di confrontare la propria produzione letteraria con quella di altri grandi autori attraverso la traduzione dei classici [...], la ricerca filologica e 
la critica testuale. Attraverso questo triplice approccio alla letteratura, egli mise a punto i fondamenti della sua poetica e della sua attività di studioso (1998: 223).

Nonostante la poetica di Foscolo non abbia ottenuto una notorietà internazionale, critici, teorici e autori come lo stesso Leopardi e Wellek considerano Foscolo uno dei più grandi rappresentanti della poesia malinconica e della discussione in corso all'epoca sulla scuola drammatica. A proposito di poeti e poesia, Leopardi, in due passi dello Zibaldone, scrive:

Quei pochissimi poeti italiani che in questo o nel passato secolo hanno avuto qualche barlume di genio e natura poetica, qualche poco di forza nell'animo [2364] o nel sentimento, qualche poco di passione, sono stati tutti malinconici nelle loro poesie (Alfieri, Foscolo ec.). (27. Gen. 1822)

[...] il linguaggio e lo stile delle poesie di Parini, Alfieri, Monti, Foscolo è molto più propriamente e più perfettamente poetico e distinto dal prosaico, che non è quello di verun altro de' nostri poeti, inclusi nominatamente i più classici e sommi antichi. (12 Setr. 1823)

Quest' idea è ripresa da Vossler quando afferma che "la clásica belleza de sus poesías está impregnada de un hálito moderno de turbadora melancolía" (1951: 147).

Per Wellek, il saggio "Della nuova scuola drammatica", che Foscolo scrisse negli ultimi anni della sua vita e nel quale criticava le teorie di Manzoni, è "the most brilliant flash of Foscolo's critical activity. The high level of the essay was never or very rarely reached before" (1970: 266).

\section{Sempre secondo l'autore di Teoria della letteratura,}

Foscolo's chief importance, especially for Italy, lies in his attempt to see the conception of poetry as part of history, of a philosophy of human development, and thus as the basis of a scheme for Italian literary history and a program for his own time. (1970: 268)

Foscolo può essere considerato colui che inaugurò la critica letteraria romantica, che privilegia l'analisi del "contenuto" rispetto a quella della "forma", e diede enfasi alla filologia, alla storia, alla psicologia ${ }^{10}$. Inoltre, anticipò due importanti linee

10. Per Vincenzo Laforgia, Foscolo fu l'iniziatore della critica psicologica. In www.repubblicaletteraria.net del 21/03/02. 
critiche dell'Ottocento, la cosiddetta critica estetica sviluppata da De Sanctis e la critica positivista e storica difesa da Carducci. Secondo Wellek,

in the history of Italian criticism Foscolo will keep an important position as the first critic who broke with neoclassicism and introduced a historical scheme for the writing and criticism of Italian literature. But in a European context Foscolo is a latercomer, an eclectic somewhere in the transition from a preclassical Platonic idealism to a romantic view of history. (1970: 272)

Per quanto riguarda la critica, Wellek considera Leopardi molto più originale e notevole di Foscolo (1970: 272). Senza dubbio, Leopardi è più originale $\mathrm{e}$ molto più autonomo nell'elaborazione della sua critica ${ }^{11}$, visto che, soprattutto nello Zibaldone, lo scrittore di Recanati formula e propone la sua propria poetica, nella quale è possibile individuare rilevanti giudizi letterari, che la critica italiana e la critica internazionale stanno mettendo in risalto con crescente frequenza negli ultimi anni.

In questo ampio "laboratorio poetico e filosofico", Leopardi getta le basi per molti progetti: filosofici, letterari, linguistici, religiosi, politici, ecc. Iris Origo sottolinea l'ampiezza degli argomenti trattati nello Zibaldone:

troviamo nello Zibaldone i germi di quasi tutti i suoi disegni letterari, non soltanto di quelli che poi diventeranno le Operette Morali o dovevano farne parte ma non furono compiuti, ma anche numerosissimi altri progetti, di cui ritroveremo frequenti elenchi nelle carte napoletane: studi grammaticali, abbozzi di tragedie, di inni, di meditazioni, di discorsi, considerazioni sulla sublimità del linguaggio della Bibbia, corsi di letteratura greca, latina, italiana, elogi degli italiani illustri, commenti sulla società italiana contemporanea e via di seguito. (1974: 186)

Abbiamo visto che sono molteplici i progetti di Leopardi, ma mi fermerò solamente ad analizzare due aspetti della sua poetica, che considero abbastanza originali

11. Per Wellek, la parola "critica" deve essere intesa in senso lato, ossia essa deve esprimere, oltre ai giudizi sui libri e aurori individualmente considerati, quello che si pensa rispetto ai principi della teoria della letreratura, alla sua natura, i suoi generi, le sue origini e la sua storia (1970a: 1). E in questo senso che uso la parola "critica". 
e che lo mettono accanto ad Aristotele e Frye, cioè: l'elaborazione di un sistema di belle arti e di una teoria dei generi letterari.

Nelle prime pagine dello Zibaldone, ci troviamo di fronte ai frammenti che contengono i tentativi di Leopardi di formulare un trattato sulle Belle Arti. Prima però di creare il suo sistema propriamente detto, Leopardi analizza gli elementi che possono fare parte di questo sistema, a partire da due questioni centrali e universali: "Qual è l'oggetto dell'arte?" e "Qual è la natura del bello?" Leopardi formula la domanda: "[...] Ora se è vero che la perfezione delle cose in sostanza consiste nel perfetto conseguimento del loro oggetto, quale sarà l'oggetto delle Belle Arti?"

Nella formulazione del suo sistema d'arte, Leopardi riflette anche sul gusto, il bello, il sublime, lo stile, i generi letterari, ecc. Apre la discussione sul suo sistema d'arte affermando: "Non il Bello ma il Vero [...] è l'oggetto delle Belle Arti" E riprendendo il concetto aristotelico d'imitazione, sottolinea che "il Vero" $\mathrm{e}$ "l'imitazione della Natura" O ancora: "La perfezione di un'opera di Belle Arti non si misura dal più Bello ma dalla più perfetta imitazione della Natura" L'imitazione, per Leopardi, è propria dell'uomo:

la facoltà imitativa è una delle principali parti dell'ingegno umano. L'imparare in gran parte non è che imitare. Ora la facolt̀̀ d'imitare non è che una facoltà di attenzione esatta e [1365] minuta all'oggetto e sue parti, e una facilità di assuefarsi. Chi facilmente si assuefa, facilmente e presto riesce ad imitar bene.

Platone, nella Repubblica, aveva anche affermato che "os poetas realizam sua narrativa por intermédio da imitação" (2000: 85). Nei primi tre capitoli della Poetica, Aristotele aveva già formulato la sua teoria d'imitazione, dicendo che la poesia è una imitazione attraverso la voce. Per questo motivo, la tragedia e la commedia imitano a partire dal ritmo, dal linguaggio e dalla melopeia. Questi risultano i punti di contatto tra i due. Ma nella sua idea sull'imitazione, Leopardi include un nuovo elemento: "l'assuefazione", un concetto ricorrente negli scritti di Leopardi e particolarmente nello Zibaldone. Per questo, Leopardi afferma: 
non solamente tutte le facoltà dell'uomo sono una facoltà di assuefarsi, ma la stessa facoltà di assuefarsi dipende dall'assuefazione. A forza di assuefazioni si piglia la facilità di assuefarsi, non solo dentro lo stesso genere di cose, ma in ogni genere.

Reiterando l'idea per cui il bello non è l'oggetto delle Belle Arti, scriverà che "Tragedia, Comedia, Satira han per oggetto il brutto ed è una mera quistion di nome il contrastar se questa sia poesia"

Leopardi, in difesa del brutto negli scritti letterari, afferma che questi sono validi se introducono una novità, associando cosi il brutto a una certa avanguardia. Per Leopardi, questo succede perché l'uomo non sopporta la noia, perciò la novità pur se brutta sarà sempre gradevole: "Perché l'uomo niente tanto odia quanto la noia, e però gli piace di veder qualche novità ancorchè brutta"

Il concetto di "novità" presente in Aristotele è ripreso da Leopardi, che lo amplifica e cos̀ anticipa le formulazioni di alcuni teorici del Novecento, più specificamente quelle dei Formalisti Russi, che considerano fondamentale la "novità" per il "materiale" e per il "procedimento"12 nella letteratura.

Dall'oggetto delle Belle Arti Leopardi ritorna al concetto d'imitazione, affermando che "La perfezione di un'opera di Belle Arti non si misura dal più Bello ma dalla più perferta imitazione della Natura", e "fonte del diletto nelle arti non è il bello, ma l'imitazione"

Se da un lato Leopardi riprende il concetto aristotelico dell' imitazione, dall'altro utilizza il concetto oraziano di "utilità" della poesia, osservando che "L'utile non è il fine della poesia benchè questa possa giovare. $\mathrm{E}$ può anche il poeta mirare espressamente all'utile o ottenerlo (come forse avrà fatto Omero) senza che però l'utile sia il fine della poesia [...]"

Dopo queste formulazioni, Leopardi cerca di definire il suo "Sistema di Belle Arti", affermando che il fine delle Belle Arti è il piacere, l'oggetto o mezzo per ottenerlo è l'imitazione della natura, la cagione primaria è la "maraviglia" e che i

12. Per informazioni più dettagliate sulla poetica dei Formalisti Russi, vedere Tomacevskij, Boris. Teoria della letterature. 
difetti nelle Belle Arti sono causati dalla sproporzione, dalla sconvenevolezza, da cose poste fuor di luogo, dall'affettazione. Nonostante questi concetti siano già stati usati da Platone, Aristotele, Orazio ed altri, Leopardi li amplia per formulare il suo proprio sistema. Haroldo de Campos lo considera "um teórico da vanguarda" (1977: 185).

Nel suo sistema, Leopardi analizza l'cessenza" della poesia, introducendo i diversi tipi d'imitazione:

Del Bello: Epopea, Lirica ec. - Del Sublime: Lirica, Epopea ec. - Del terribile: Tragica ec. - Del ridicolo e vizioso ec.: Commedia Satira poesia bernesca ec. - Vari rami del bello. Bello delicato - grazioso - ameno - elegante. V. MARTIGNONI ec. - Annali di Scienze e Lettere, n. 8, p. 252-54. Ci può essere il bello delicato e il non delicato. Ercole, Apollo. Bello Sublime. Giove.

Implicitamente, l'autore delle Operette morali stabilisce una sua teoria dei generi, incentrata su una visione triplice: lirico, epico e drammatico. È da sottolineare che il genere, uno dei temi più discussi nella teoria della letteratura, sarà teorizzato da Leopardi nello Zibaldone.

Per gli antichi, la nozione di genere era inseparabile dallo stile, il quale era diviso in semplice, medio e sublime. Leopardi inverte questa nozione e parte dallo stile per giungere al genere, perché è dentro al "bello", al "sublime", al "terribile" e al "ridicolo" che lo scrittore italiano inserisce, in un primo momento, i generi letterari. Questa "inversione" di Leopardi sembra avvicinarsi ad una delle formulazioni di Compagnon quando afferma che lo stile

recouvre également beaucoup d'autres aspects du discours, non seulement d'autres traits formels qu'on a généralement cessé d'amalgamer au style (comme le genre: un texte exemplifie le genre auquel il appartient), mais aussi des aspects relevant du contenu, et même de la substance du contenu. (1998: 226)

Per questo, sia lo stile sia il genere non si inseriscono in una definizione precisa; sono nozioni complesse, ricche, ambigue, molteplici. Successivamente, Leopardi aggiunge al suo sistema di Belle Arti un altro elemento di stile: la "semplicità" Questa è quasi sempre legata alla bellezza, al buon gusto e alla naturalezza, perché 
la semplicità è quasi sempre bellezza sia nelle arti, sia nello stile, sia nel portamento, negli abiti ec. ec. ec. Il buon gusto ama sempre il semplice. [...] La semplicità è bella, perché spessissimo non è altro che naturalezza; cioè si chiama semplice una cosa, non perch'ella sia astrattamente e per se medesima semplice, ma solo perché è naturale, non affettata, non artifiziata, semplice in quanto agli uomini, non a se stessa, e alla natura ec.

Le riflessioni di Leopardi sulla semplicità appaiono in diversi passi dello $Z i$ baldone e non sono solo un elemento molto importante nelle concezioni teoriche dell'autore delle Operette morali, ma fanno parte anche della sua pratica. Generalmente, questo tipo di approccio teorico e pratico avvicina lo scrittore di Alla Luna agli antichi, specialmente ai greci, e lo allontana dalla avanguardia.

Quando propone il suo sistema di Belle Arti, Leopardi discute la questione del genere letterario, che è ripresa in diversi frammenti, formando un'ampia teorizzazione su questo argomento.

Leopardi affronta la discussione sui generi letterari mediante la tripartizione:

La poesia, quanto a' generi, non ha in sostanza che tre vere e grandi divisioni: lirico, epico e drammatico. Il lirico, primogenito di tutti; proprio di ogni nazione anche selvaggia; più nobile e più poetico d'ogni altro [...]. L'epico nacque dopo questo e da questo; non è in certo modo che un'amplificazione del lirico [...]. Il drammatico è ultimo dei tre generi, di tempo e di nobiltà. Esso non è un'ispirazione, ma un'invenzione; figlio della civiltà, non della natura; poesia per convenzione e per volontà degli autori suoi, più che per la essenza sua [...]. Gli altri che si chiamano generi di poesia, si possono tutti ridurre a questi tre capi, o non sono generi distinti per poesia, ma per metro o cosa tale estrinseca. (Recanati, 15 Dicembre 1826)

Apparentemente, seguendo in parte la tradizione del classicismo, e diversamente dai greci e latini, Leopardi dà importanza ad un genere che fino ad allora non era stato contemplato, la lirica. La gerarchia dei generi proposta da Leopardi, postulando il lirico come un genere superiore, lo avvicina alla tradizione classica e lo allontana $\mathrm{da}$ Aristotele, quando questi afferma che la tragedia è il genere più nobile.

Leopardi realizza un' inversione completa dei generi letterari, attribuendo al lirico lo statuto di "primogenito" di turti i generi. In questo modo, si allontana dalle poe- 
tiche greche e latine, come pure dalle formulazioni del classicismo, che esaltavano la tragedia e l'epica, e da alcune concezioni del romanticismo. Le osservazioni di Leopardi sulla superiorità del genere lirico si trovano in molti passi dello Zibaldone, come nel frammento in cui sostiene che "la lirica si può chiamare la cima il colmo la sommità della poesia, la quale è la sommità del discorso umano"

Come si può vedere, la lirica è il genere per eccellenza per l'autore dei Canti. Nello Zibaldone un grande spazio è dedicato alla discussione del genere lirico a sfavore di altri generi. Per questo Wellek mostra che

the emphasis on the lyric which we have mer in Herder or Foscolo becomes much more startling in Leopardi when we see him draw the consequences and disparage the epic and the drama. This was far from the thought of Herder, who at most wanted to assimilate all genres into one. Leopardi makes similar attempts to deny the distinction between the lyrical and the epic [...]. The drama comes off even worse. It belongs even less to poerry than does the epic. (1970: 276-7)

Se Wellek non avesse rivolto solamente l'attenzione alla teoria leopardiana della lirica, avrebbe percepito che Leopardi ha formulato anche un'ampia teoria del dramma. Ma nello Zibaldone è possibile trovare quello che Wellek ha denominato

[...] the most complete reversal of the neoclassical hierarchy of genres. Drama and plot, which in Aristotle are the essence of poetry, are banned by Leopardi to its periphery. The lyric, which had been excluded by Bacon and Hobbes from poetry, and the expression of personal feeling, are the only poetry, the highest kind. The wheel has come to a full circle. (1970: 278)

Per Wellek il cerchio si chiude con la teoria della lirica leopardiana e cos̀̀ egli innalza Leopardi nella schiera dei grandi teorici come Platone e Aristotele.

Tuttavia potremmo rielaborare questa affermazione dicendo che il cerchio che si chiude con Leopardi in realtà si trasforma in un'enorme spirale, dato che i generi letterari ritornano ad essere ampiamente studiati dai critici e teorici, soprattutto a partire dalla seconda metà del Novecento ${ }^{13}$. La teoria e la critica attribuiscono al

13. Durante la prima metà del sec. $\mathrm{XX}$, sotto l'influenza di Croce, si è diffuso in molti settori della critica e della ricerca letreraria un forte scerricismo rispetto al concerto di genere. 
concetto di genere un ruolo rilevante, come dimostrano, ad esempio, Frye, Staiger, Genette, Käte Hamburger e Compagnon.

Nelle sue riflessioni sui generi e sul sistema di Belle Arti, Leopardi dialoga con le poetiche classiche, rinascimentali, neoclassiche e romantiche, e sviluppa una concezione propria del genere letterario, che influenzerà le poetiche future.

Con la sua formulazione sulla lirica, Leopardi prepara e anticipa una concezione di poesia che sarà ripresa da diversi teorici, tra i quali Poe, Eliot, Pound, i Formalisti Russi e, nella pratica, sarà recuperata dai poeti ermetici italiani.

\section{Poetiche a confronto}

Dopo aver analizzato alcuni aspetti della poetica di Foscolo e di Leopardi, è possibile dire che uno dei punti convergenti tra i due poeti è l'uso del frammento. Il frammento è la base di composizione di alcune opere di Foscolo, come Le ultime lettere di Jacopo Ortis, Dei Sepolcri e Le Grazie. Secondo The Cambridge History of Italian Literature:

Le Grazie was allegedly inspired by Greek poetic fragments; that it was begun soon after Ortis, itself structured like a series of epistolary fragments; that artistic sensibility had been for some time influenced by archaeological discoveries leading to the appreciation of the aesthetic possibilities not merely of incomplete works found as fragments, but of art, even criticism, initially conceived or offered as fragmentary. (1999: 414)

Il frammento viene anche utilizzato da Leopardi nel suo Zibaldone di Pensieri ${ }^{14}$. Nonostante sia un'opera apparentemente non sistematica, esiste qualcosa di coerente nello Zibaldone, che forma un solido sistema di idee e concetti, presentando una coerenza interna nella esposizione delle sue riflessioni.

14. Secondo Haroldo de Campos, Leopardi "antecipa [...] a poérica do fragmentário, que hoje, dentro da poesia italiana, reencontramos levada às suas conseqüências estilísticas exatas na obra ungarettiana" (1977: 90). 
Se da un lato l'uso del frammento era una pratica del passato che poi sarà ripresa dai moderni, dall'altro permette che Foscolo e Leopardi siano considerati scrittori in linea con il loro tempo, come molti dei romantici tedeschi.

Si sottolinea inoltre che Foscolo e Leopardi dimostrano un grande interesse per gli antichi, in special modo i greci. E una questione che interessa entrambi è, ad esempio, il dibattito antico versus moderno.

Questa dicotomia antico versus moderno, molto discussa durante il Settecento e anche l'Ottocento, è presente sia nel romanzo epistolare Le ultime lettere di Jacopo Ortis di Foscolo quando, ad esempio, il protagonista dice "beati gli antichi che si credevono degni de' baci delle immortali dive del cielo... e che trovavano il Bello e il Vero accarezzando gli idoli della lor fantasia"15, sia nel commento alla traduzione di La chioma di Berenice, che secondo i curatori di The Cambridge History of Italian Literature presenta

the substancial difference between the ancient and the modern world, raising all manner of issues, concerning not only the function of poetry and literature in either, or both, but also the possibility of transposing poetic forms and contents from the distant past to present, as well as from one language to another. (1999: 415)

È anche nel saggio "Dell'origine e dell'ufficio della letteratura" che Foscolo dimostra il suo amore per i greci, quando afferma che

[...] la letreratura greca fu sorgente ed esempio agli studj di tutta l'Europa, perché niun popolo trapassò veloce al pari degli Ateniesi dalla fierezza della barbarie alla raffinatissima civiltà; e niuno potè riunire, quant'essi, le passioni e il criterio, che pur sogliono preponderare ad età differenti negl' individui, ne' popoli e nelle lingue. (1995: 524)

Le riflessioni di Leopardi su questo tema si trovano nel saggio "Discorso di un italiano intorno alla poesia romantica" (1818) e in molti frammenti dello Zibaldone.

15. Vedere Le ultime lettere di Jacopo Ortis in http://www.fausernet.novara.it/fauser/biblio/index012.htm dell' $11 / 03 / 02$. 
Inoltre dे possibile dire che la produzione letteraria foscoliana si divide in due linee, la neoclassica e la preromantica. Ne L'Enciclopedia della letteratura De Agostini, Foscolo è considerato "la figura più significativa della letteratura italiana del periodo napoleonico. Fu, insieme, interprete della poetica della classicità e spirito fortemente romantico" (1997: 374). Già in The New Princeton Encyclopedia of Poetry and Poetics si legge che Foscolo "fuses the classicist's love for perfection of form with the heritage of Parini and Alfieri and with European romanticism" (1993: 645).

Per quanto riguarda Leopardi, Sainte-Beuve dice "Leopardi appartient à l'école des novateurs, il était du moins le classique par excellence entre les romantiques" (1994: 37). Sebbene Leopardi abbia difeso una letteratura non romantica in Lettera ai compilatori della "Biblioteca italiana" in risposta a quella di Madama la baronessa di Stäel (1816) e nel Discorso di un italiano intorno alla poesia romantica (1818), o ancora in alcune parti dello Zibaldone, una minuziosa analisi della sua opera rivelerà un Leopardi che presenta a volte caratteristiche del classicismo, a volte del romanticismo. É possibile scorgere una relazione dialettica, che può avere come sintesi la mescolanza di caratteristiche dei due periodi. $\mathrm{O}$ ancora, come abbiamo detto, la grandezza di Leopardi si colloca fuori dal tempo, confermando quello che ha dichiarato Luporini: "l'esperienza leopardiana ha le sue radici essenzialmente nell'epoca romantica, ma tuttavia la oltrepassa" (1996: 5).

In generale, la critica ha una certa difficoltà nello stabilire a che epoca letteraria Leopardi appartenga, neoclassica o preromantica o classica e romantica. Sono questioni che servono per capire meglio, situare e confrontare le poetiche di Foscolo e Leopardi, perché, come sottolinea Wellek, Foscolo e Leopardi "who expressly attacked the theories of the romantic group, themselves best represent the turn in Italy toward doctrines which were the basis of European romanticism" (1970: 265).

Oltre alla componente frammentaria e alla valorizzazione degli antichi a sfavore dei moderni, un altro elemento che avvicina le poetiche di entrambi è la valorizzazione della parola. In Foscolo questo aspetto è implicito in "Dell'ufficio e dell'origine della letteratura" e nella sua analisi di alcune traduzioni. Per Leopardi, la preoccupazione 
per la parola è centrale, sia nei suoi studi di filologia, sia nella critica, teoria e pratica della traduzione, sia nell'interesse per il verso ben fatto e per la prosa accurata.

Per quanto riguarda la valorizzazione della parola, è possibile dire che la loro attività di traduttori è un aspetto "moderno" di entrambi i poeti, perché dimostra un'apertura verso l'esterno, ossia la coscienza del fatto che era necessario un rinnovamento attraverso l'importazione di modelli di eccellenza, dei moderni e degli antichi secondo Foscolo, degli antichi secondo Leopardi.

Un elemento che li allontana è che Foscolo, in alcuni sonetti e carmi e nel romanzo epistolare, sviluppa la poetica dell'esilio, la quale si collega al passato. Invece Leopardi elabora nello Zibaldone una poetica dell'infinito, del vago, dell'indefinito, che si collega con il futuro.

Altra questione dissonante tra i due è che Leopardi teorizza sui generi letterari, mentre Foscolo, come Croce, li rigetta. Secondo Wellek

Foscolo even rejects all genre and school distinctions, saying that "every great production is an individual object which has different merits and distinct characteristics". He protests against the lumping together of different, supposedly classical schools of drama: the Greeks, the French, and the Italians (Alfieri). (1970: 266)

\section{Conclusione}

Per concludere, è possibile dire che la poetica di Leopardi è più universale, perché si preoccupa di più degli aspetti estetici, che oltrepassano il suo tempo. Leopardi è molto più indipendente, autonomo e innovatore, perché mentre si rivolge al passato postula una nuova modernità; per questo motivo non si inserì nelle correnti intellettuali e nei modi di scrivere dominanti nel suo Paese.

A sua volta, Foscolo sviluppò una poetica che definirei "schierata", perché molto impegnata nella politica e negli aspetti sociali della sua epoca e, per questo, meno universale e più nazionale. Forse per questo Walter Binni propone di interpretare l'opera di Foscolo a partire da una visione più storica, considerando che 
mentre la sua poetica si collega vitalmente a tutta la sua Weltanschaung, al suo pensiero politico, alla sua esperienza e sofferenza storica, alla sua complessa cultura e attraverso queste ai problemi e alle condizioni della sua epoca irrequieta e feconda, essa si alimenta di un vivo dialogo con le esperienze letterarie e con le poetiche generali e personali del suo tempo. (1993: 25)

Forse mancò alla poetica di Foscolo quel "vigore d'intelletto" che De Sanctis descrisse quando parlò della sua prosa (1996: 784).

È ancora possibile, infine, affermare che Foscolo ̀̀ paragonabile a José de Alencar, come Leopardi a Machado de Assis. Il primo è nazionale, l'altro internazionale. Nella poetica di Foscolo, come in quella di Alencar, è presente un forte interesse per un progetto nazionale, politico-culturale, $e$ in quella di Leopardi predomina una preoccupazione estetica e quindi più universalizzante $e$ atemporale.

Inoltre, la poetica di Foscolo si affianca all'estetica platonica, che tende a non dare importanza all'arte come diversità e molteplicità. La poetica di Leopardi, al contrario, si avvicina all'estetica aristotelica, che concede un'accurata attenzione alle distinzioni che sono necessarie nel dominio dell'arte, in generale, e nel dominio della poesia, in particolare, analizzando i testi poetici nella loro diversità empirica.

Credo che mediante ricerche e studi più accurati sulla poetica di Foscolo e di Leopardi e con una maggior quantità di traduzioni della loro opera in altre lingue, sia possibile far sì che questi due grandi della letteratura italiana diventino un riferimento nella critica e nella teoria letteraria dell'Occidente.

RESUMO: Apds analisar e comparar alguns aspectos da poetica de Foscolo e Leopardi, é possivel afirmar que a pottica de Leopardi é mais universal, porque se preocupa mais com aspectos estéticos, que ultrapassam o seu tempo. Já a poética de Foscolo é mais nacional, porque mais preocupada com aspectos politicos e sociais de sua época.

PALAVRAS-CHAVE: literatura italiana; Foscolo; Leopardi. 


\section{Referenze bibliografiche}

ARISTOTELE. Poetica, traduzione e introduzione di Guido Paduano, Bari, Laterza, 1998.

BINNI, Walter, La nuova poetica leopardiana, Milano, Sansoni, 1997.

, Poetica, critica e storia letteraria, Firenze, Le Lettere, 1993.

BORGES, Jorge Luis e WILDE Eduardo, El idioma de los argentinos, Barcelona, Seix Barral, 1997.

CAMPOS, Haroldo de, $A$ arte no borizonte do provdvel, São Paulo, Perspectiva, 1977 ( $4^{\mathrm{a}} \mathrm{ed}$.)

CAPRIO, Vincenzo de \& GIOVANARDI, Stefano, I testi della letteratura italiana: l'Ottocento, Milano,

Einaudi Scuola, 1998.

CARPEAUX, Otto Maria, Ensaios reunidos, 1942-1978, Rio de Janeiro, Topbooks, 1999, vol. I.

História da Literatura Ocidental, Rio de Janeiro, O Cruzeiro, 1959 a 1966, 7 v.

CESERANI, R. \& FEDERICIS, L. De, Il materiale e l'immaginario, Torino, Loescher, 1993, v. 4.

COMPAGNON, Antoine, Le Démon de la theorie. Littérature et sens commun, Paris, Seuil, 1998.

CROCE, Benedetto, Aesthetica in nuce, Bari, Laterza e Figli, 1946.

, Letrure di poeti, Bari, Laterza e Figli, 1966.

, Problemi di estetica e contributi alla storia dell'estetica italiana, Bari, Laterza e Figli, 1954 ( $5^{\text {a }}$ ed.).

DE SANCTIS, Francesco, Giacomo Leopardi, Roma, Editori Riuniti, 1983.

, Opere, Milano/Napoli, Riccardo Ricciardi Editore, 1986.

, Storia della Letteratura italiana, Torino, Einaudi-Gallimard, 1996.

DONADONI, Eugenio, Biografia di Ugo Foscolo, Napoli, Francesco Perrella, 1999. In http://www. fausernet.novara,it/fauser/biblio/index130.hem del 01/03/02.

EYNAUD, Joseph, "Ugo Foscolo traduttore di Omero e di Laurence Sterne", in www.repubblicaletteraria.net del 21/03/02.

FOSCOLO, Ugo, Frammenti abbozzati. Della ragione poetica. Del sistema e dell'architettura del carme.

In hrtp://www.fausernet.novara.it/fauser/biblio/index130.htm dell'11/03/02.

, Opere. Prose e saggi, Milano, Einaudi/Gallimard, 1995, vol. II.

GUERINI, Andréia, A poética de Leopardi: gênero e tradufăo no Zibaldone di Pensieri, Florianópolis, Tese de doutorado, 2001 (in corso di stampa).

HOBSBAWN, Eric J., A era das revolufōes - Europa 1789-1848, trad. Maria Tereza Lopes Teixeira e

Marcos Penchel, Rio de Janeiro, Paz e Terra, 2001 (13a. ed.).

LAFORGIA, Vincenzo, "Ugo Foscolo critico letterario" in www.repubblicaletteraria.net del 21/03/02.

LEOPARDI, Giacomo, Canti, Milano, Rizzoli, 1998.

,Operette Morali, Milano, Biblioteca Universale Rizzoli, 1996. 
LEOPARDI, Giacomo, Zibaldone di Pensieri, Milano, Mondadori, 1983. , Zibaldone, Roma, Grandi Tascabili Economici Newton, 1997.

, Zibaldone di Pensieri, in http://www.liberliber.it/biblioteca/1/leopardi/ del 02/02/01.

Poesie e Prose, a cura di Mario Andrea Rigoni, Milano, Arnoldo Mondadori / Meridiani,

1998. Volume primo: Poesie.

LUPORINI, Cesare, Leopardi Progressivo, Roma, Ed. Riuniti, 1996.

ORIGO, Iris, Leopardi, traduçāo de Paola Ojetti, Milano, Rizzoli Editore, 1974.

PAZZAGLIA, Mario, Letteratura italiana. L'Ottocento. Testi e critica con lineamenti di storia letteraria,

Bologna, Zanichelli, 1991, v. 3.

PLATĀO, A Repuiblica, trad. Enrico Corvisieri, São Paulo, Nova Cultural, 2000.

RIENZO, Giorgio de, Breve Storia della Letteratura Italiana, Milano, Bompiani, 1997.

SAINTE-BEUVE, Charles A., Portrait de Leopardi, Paris, Allia, 1994.

SALINARI, C. e RICCI, C., Storia della letteratura italiana, Roma-Bari, Laterza, 1986, v. 3.

SQUAROTTI, Giorgio B., Letteratura Italiana. Lineamenti. Problemi. Autori, Messina-Firenze, G. D'Anna, 1991.

STAIGER, Emil, Conceitos fundamentais da poética, trad. Celeste Aída Galeāo, Rio de Janeiro, Tempo Brasileiro, 1975.

STERNE, Laurence, Viaggio sentimentale di Yorick lungo la Francia e l'ltalia, trad. Ugo Foscolo, Milano, Garzanti, 1998.

TOMACEVSKIJ, Boris, Teoria della Letteratura, Milano, Feltrinelli, 1978.

UNGARETTI, Giuseppe, Razōes de uma poesia, trad. Liliana Laganà, Lucia Wataghin, Maria Betânia Amoroso, Sāo Paulo, Edusp \& Imaginário, 1994.

VOSSLER, Karl, Historia de la literatura italiana, trad. Manuel de Montoliu, Barcelona, Editorial Labor, 1951.

WELLEK, René, A History of Modern Criticism (1750-1950), London, Lowe and Brydone, 1970, 4 v. 